\title{
Erratum to: Development of amultiplex PCR for identification of Dictyocaulus lungworms in domestic and wild ruminants
}

\author{
Anna M. Pyziel ${ }^{1} \cdot$ Zdzislaw Laskowski $^{1} \cdot$ Johan Höglund $^{2}$
}

Published online: 1 September 2015

(C) Springer-Verlag Berlin Heidelberg 2015

\section{Erratum to: Parasitology Research}

DOI 10.1007/s00436-015-4657-y

The original version of the article unfortunately contained a mistake. The published sequence of the species-specific forward primer for Dictyocaulus viviparus is incomplete. The last 5 nucleotides are missing from the $3^{\prime}$ terminus of the primer.

Accordingly, the sentence "These included three species-specific forward primers for D. viviparus (5'GCTAATGTTCTTAATCGT-3') located 191 bases upstream of the $3^{\prime}$ end of the ITS2 region, D. eckerti (5'CTATATGGAAATGATGATTACCGT-3') located 351 bases upstream of the $3^{\prime}$ end of the ITS2 of D. eckerti and 291 bases upstream of $3^{\prime}$ end of the ITS2 of $D$. sp. from red deer and moose, and finally, D. capreolus (5'-CGAGATACATGTCATATCGA-3') located 30 bases upstream of the $3^{\prime}$ end of the ITS2, as well as one reverse primer

(5'-CACAGTACTTGTTTGCTATCG-3') located approximately 363 bases downstream of the $5^{\prime}$ end of the $28 \mathrm{~S}$ rRNA gene, which was common for all lungworm species examined" should be corrected as follows:

Corrected Sentence

These included three species-specific forward primers for D. viviparus (5'-GCTAATGTTCTTAATCGTTGATC-3') located 191 bases upstream of the $3^{\prime}$ end of the ITS2 region, D. eckerti (5'-CTATATGGAAATGATGATTACCGT-3') located 351 bases upstream of the $3^{\prime}$ end of the ITS2 of D. eckerti and 291 bases upstream of $3^{\prime}$ end of the ITS2 of $D$. sp. from red deer and moose, and finally, D. capreolus (5'-CGAGATACATGTCATATCGA-3') located 30 bases upstream of the $3^{\prime}$ end of the ITS2, as well as one reverse primer (5'-CACAGTACTTGTTTGCTATCG-3') located approximately 363 bases downstream of the $5^{\prime}$ end of the 28S rRNA gene, which was common for all lungworm species examined.

The online version of the original article can be found at http://dx.doi.org/ 10.1007/s00436-015-4657-y.

Anna M. Pyziel

anpyz@twarda.pan.pl

1 W. Stefański Institute of Parasitology, Polish Academy of Sciences, Twarda 51/55, 00-818 Warsaw, Poland

2 Department of Biomedical Sciences and Veterinary Public Health, Section of Parasitology, Swedish University of Agricultural Sciences, 75007 Uppsala, Sweden 